11-1-2009

\title{
A Linear B-Spline Threshold Dose-Response Model with Dose-Specific Response Variation Applied to Developmental Toxicity Studies
}

Chin-Shang Li

University of California, Davis, cssli@ucdavis.edu

Daniel L. Hunt

Radiation Therapy Oncology Group, Philadelphia, PA, dhunt@acr.org

Follow this and additional works at: http://digitalcommons.wayne.edu/jmasm

Part of the Applied Statistics Commons, Social and Behavioral Sciences Commons, and the Statistical Theory Commons

\section{Recommended Citation}

Li, Chin-Shang and Hunt, Daniel L. (2009) "A Linear B-Spline Threshold Dose-Response Model with Dose-Specific Response Variation Applied to Developmental Toxicity Studies," Journal of Modern Applied Statistical Methods: Vol. 8 : Iss. 2 , Article 22. DOI: $10.22237 /$ jmasm/1257034860

Available at: http://digitalcommons.wayne.edu/jmasm/vol8/iss2/22 


\title{
A Linear B-Spline Threshold Dose-Response Model with Dose-Specific Response Variation Applied to Developmental Toxicity Studies
}

\author{
Chin-Shang Li \\ Daniel L. Hunt \\ University of California, Radiation Therapy Oncology Group, \\ Davis \\ Philadelphia, PA
}

A linear $B$-spline function was modified to model dose-specific response variation in developmental toxicity studies. In this new model, response variation is assumed to differ across dose groups. The model was applied to a developmental toxicity study and proved to be significant over the previous model of singular response variation.

Key words: Developmental toxicity study, dose-response, interior knot, linear $B$-spline, response variation, threshold.

\section{Introduction}

In a developmental toxicity study, fetal response is measured and recorded in each litter of an animal that has been directly exposed to some toxic substance that is environmentally ambient and that poses a developmental threat. Developmental endpoints include death, abnormality (all types), weight and length. A positive fetal response, equivalent to negative indicators of these endpoints, implies negative reaction to the toxic substance. Upon study execution, the fetal risk of indirect exposure can be assessed. The U.S. Environmental Protection Agency (USEPA) uses such study results to determine safety exposure levels for the general population (USEPA, 1991); statistical modeling is a key factor in estimating risk (Ryan, 2000).

The default assumption in the risk assessment process for developmental toxicity studies is that a threshold dose level exists

Chin-Shang Li is an Associate Professor in the Division of Biostatistics, Department of Public Health Sciences, University of California, Davis, CA. Email: cssli@ucdavis.edu. Daniel L. Hunt is a Senior Statistician in the RTOG Statistical Center, American College of Radiology, Philadelphia, PA. Email: dhunt@acr.org.
(USEPA, 1991). Threshold is the maximum dose level at which the response is equivalent to the background response. The USEPA uses the no-observed-adverse-effects-level (NOAEL) and benchmark dosing approaches. The NOAEL approach identifies the highest dose level at which the response is not statistically significant from the control. Benchmark dosing employs actual dose-response modeling. First proposed by Crump (1984), the benchmark dose is a lower confidence limit for the dose equivalent to a level that yields an acceptable limit excess risk. Although both approaches search for a tolerable dose level, neither is a pure threshold model.

Cox (1987) introduced a variety of pure threshold models for application to toxicology studies. Schwartz, et al. (1995) applied a threshold model to a developmental toxicity study, using quasi-likelihood techniques for estimating model parameters. Hunt and Rai (2003) introduced the threshold dose-response model with a single parameter for response variation included in the dose-response function. All these approaches model the behavior of the dose-response pattern below the threshold level as one of constant response. The model proposed in this study inherently estimates the threshold, while tracking the change in the slope of the dose-response curve, thereby allowing more flexibility in the sense of being able to model multiple dose-response shapes. 
Hunt and Rai (2003) first modeled the observed variation by including a parameter that equated to the interlitter response variation. Subsequently, they modified their model to include additional parameters to account for the noticeable multiple response variation across dose groups (Hunt \& Rai, 2007). They reported significance with the model of dose-specific response variation compared to a model with uniform variation across dose levels. They also conducted simulations and found that the dosespecific model with multiple variation parameters led to unbiased estimation of all model parameters, whether the true variance structure was that of single or multiple parameters, whereas the single-parameter model was less robust. These results are similar to those of Kupper, et al. (1986), who assumed the beta-binomial distribution for the response number for each litter and a logistic doseresponse model and found that the model with the multiple-intralitter correlation structure produced less biased results than the model that assumed a single-intralitter correlation.

Similar to Hunt and Rai (2007), the model used in this study includes dose-specific parameters that estimate the response variation in addition to using polynomial regression splines to fit to the dose-response pattern (Ramsay, 1988). The regression spline approach was used formerly in a model with one parameter for response variation (Li \& Hunt, 2004). As the polynomial, degree one (linear) was used and a set of $B$-splines was constructed recursively to help fit the model. The theory of $B$-splines is described in de Boor (2001). Integral to this theory is the incorporation of interior knots as change points in the direction of the plotted curve. The ability to incorporate these knots is desirable for data from developmental studies as the threshold is inherently assumed.

\section{Methodology}

In a developmental toxicity study, there are $g$ dose groups, each of which has a certain level of a toxic substance. The $i^{\text {th }}$ dose group contains $m_{i}$ animals, and therefore litters $(i=1, \ldots, g)$. For $n_{i j}$ implantations of the $j^{\text {th }}$ animal $(j=1, \ldots$, $m_{i}$ ) in the $i^{\text {th }}$ dose group, let $x_{i j}$ be the number of fetuses that experience at least one adverse effect. Adverse effects include early and late fetal death and any kind of malformation (morphological, visceral or skeletal). An adverse effect such as death supersedes malformation. If $P_{j}\left(d_{i}\right)$ be the probability of a fetus in the $j^{\text {th }}$ litter indirectly exposed to the $i^{\text {th }}$ dose level, $d_{i}$, experiencing an adverse event, then the proposed dose-response model is the following:

$$
\begin{aligned}
P_{j}\left(d_{i}\right) & =\frac{\exp \left(\sum_{k=1}^{3} \theta_{k} B_{k, 2}\left(d_{i}, \xi\right)+\sigma_{i} z_{i j}\right)}{1+\exp \left(\sum_{k=1}^{3} \theta_{k} B_{k, 2}\left(d_{i}, \xi\right)+\sigma_{i} z_{i j}\right)} \\
& =\frac{\exp \left(\mathbf{B}_{2}\left(d_{i}, \xi\right) \boldsymbol{\theta}+\sigma_{i} z_{i j}\right)}{1+\exp \left(\mathbf{B}_{2}\left(d_{i}, \xi\right) \boldsymbol{\theta}+\sigma_{i} z_{i j}\right)}
\end{aligned}
$$

Here,

$$
\mathbf{B}_{2}\left(d_{i}, \boldsymbol{\xi}\right)=\left(B_{1,2}\left(d_{i}, \boldsymbol{\xi}\right), B_{2,2}\left(d_{i}, \boldsymbol{\xi}\right), B_{3,2}\left(d_{i}, \boldsymbol{\xi}\right)\right)
$$

is the set of (order 2, degree 1) linear $B$-splines, with 1 interior knot, $\xi$, defined on the dose interval $\left[d_{1}, d_{g}\right)$, and derived recursively from the order 1 (degree 0) $B$-splines $B_{k, 1}\left(d_{i}, \xi\right)$. If $\xi_{1}=\xi_{2}=d_{1}, \xi_{3}=\xi$, and $\xi_{4}=\xi_{5}=d_{g}$, then, the order $1 B$-splines are given by:

$$
B_{k, 1}\left(d_{i}, \xi\right)=\left\{\begin{array}{ll}
1, & d_{i} \in\left[\xi_{k}, \xi_{k+1}\right) \\
0, & \text { otherwise }
\end{array} k=1,2,3,\right.
$$

and the order $2 B$-splines formed recursively from (2) are given by:

$$
\begin{aligned}
B_{k, 2}\left(d_{i}, \xi\right)= & \frac{d_{i}-\xi_{k}}{\xi_{k+1}-\xi_{k}} B_{k, 1}\left(d_{i}, \xi\right) \\
& +\frac{\xi_{k+2}-d_{i}}{\xi_{k+2}-\xi_{k+1}} B_{k+1,1}\left(d_{i}, \xi\right)
\end{aligned}
$$




\section{LI \& HUNT}

Also from (1), the number of elements in the three-parameter vector $\boldsymbol{\theta}=\left(\theta_{1}, \theta_{2}, \theta_{3}\right)^{T}$, for the linear $B$-splines, corresponds to the degree, 1, plus the order, 2 (see de Boor, 2001). The interior knot $\xi$ represents a change point in the direction of the dose-response relationship. The modification in equation (1) from the previous model is in the parameter(s), $\sigma_{i}$, coefficients of $z_{i j} \sim N(0,1)$. Thus, with the subscript $i$, the response variability $\sigma_{i}^{2}$ is allowed to differ across dose levels $i=1, \ldots, g$.

The likelihood function based on the dose-response model in equation (1) is given by:

$$
\begin{aligned}
& L(\boldsymbol{\theta}, \boldsymbol{\sigma}, \boldsymbol{\xi})= \\
& \quad \prod_{i=1}^{g} \prod_{j=1}^{m_{i}}\left(\begin{array}{l}
n_{y_{j}} \\
x_{j}
\end{array}\right) \int_{-\infty}^{\infty} P_{j}^{x_{j}}\left(d_{i}\right)\left[1-P_{j}\left(d_{i}\right)\right]^{n_{j}-x_{j}} \frac{\exp \left(-z_{i j}^{2} / 2\right)}{\sqrt{2 \pi}} d z_{i j}
\end{aligned}
$$

where $\boldsymbol{\sigma}=\left(\sigma_{1}, \ldots, \sigma_{g}\right)$. Also note, the interior knot $\xi$ is regarded as a parameter of the model that must be estimated in addition to the parameters $\boldsymbol{\theta}$ and $\boldsymbol{\sigma}$. The likelihood function in equation (4) integrates out the random effect $z_{i j}$ from the joint distribution, thereby leaving a marginal function for the number of fetal responses $x_{i j}$ (see Collett 1991, p. 208).

Because (4) cannot be solved directly, an approximation is used via the Gauss-Hermite formula for numeric integration, given by:

$$
\int_{-\infty}^{\infty} f(u) \exp \left(-u^{2}\right) d u=\sum_{l=1}^{q} a_{l} f\left(b_{l}\right)
$$

Here $q$, the values of which $a_{l}$ and $b_{l}$ depend, is chosen to approximate (5). The standardized tables from which the values of $q, a_{l}$, and $b_{l}$ may be found are in Abramowitz and Stegun (1972).

To approximate, first let $z_{i j}=u \sqrt{2}$, based on equation (5), take the $\log$ of the likelihood function in equation (4), and approximate the log-likelihood function by:

$$
\begin{aligned}
& \ell(\boldsymbol{\theta}, \boldsymbol{\sigma}, \boldsymbol{\xi}) \approx \\
& \quad-\frac{N}{2} \times \log \pi+\sum_{i=1}^{g} \sum_{j=1}^{m_{i}} \log \left(\begin{array}{l}
n_{i j} \\
x_{i j}
\end{array}\right)+\tilde{\ell}(\boldsymbol{\theta}, \boldsymbol{\sigma}, \boldsymbol{\xi}),
\end{aligned}
$$

where $N=\sum_{i=1}^{g} \sum_{j=1}^{m_{i}} n_{i j}$ the study sample size, and

$$
\begin{aligned}
& \tilde{\ell}(\boldsymbol{\theta}, \boldsymbol{\sigma}, \boldsymbol{\xi})= \\
& \sum_{i=1}^{g} \sum_{j=1}^{m_{j}} \log \left(\sum_{l=1}^{q} a_{l} \frac{\left[\exp \left(\sum_{k=1}^{3} \theta_{k} B_{k, 2}\left(d_{i}, \boldsymbol{\xi}\right)+\sigma_{i} b_{l} \sqrt{2}\right)\right]^{x_{j}}}{\left[1+\exp \left(\sum_{k=1}^{3} \theta_{k} B_{k, 2}\left(d_{i}, \xi\right)+\sigma_{i} b_{l} \sqrt{2}\right)\right]^{n_{j}}}\right)
\end{aligned}
$$

A value of $q=20$ was chosen for the approximation; this value has been deemed acceptable in many settings (Collett, 1991).

A profile-likelihood approach was used to maximize $\tilde{\ell}(\boldsymbol{\theta}, \boldsymbol{\sigma}, \boldsymbol{\xi})$ in equation (7) and to estimate the parameters of the model. The approach begins with a search over the dose interval $\left(0, d_{g}\right)$, the domain of $\xi$. A large number of $G$ grid points $\left\{\xi_{t}^{*}: t=1, \ldots, G\right\}$ were chosen by using the formula: $\xi_{t}^{*}=d_{g} \times t /(G+1)$. Once a fixed grid point $\xi_{t}^{*}$ was selected, the order 1 and $2 B$-splines in equations (2) and (3) were calculated by regarding the fixed grid point $\xi_{t}^{*}$ as the interior knot for that part of the search. As a result, the maximizer of the profile-likelihood $\tilde{\ell}\left(\boldsymbol{\theta}, \boldsymbol{\sigma}, \xi_{t}^{*}\right)$, denoted by $\left(\hat{\boldsymbol{\theta}}_{t}, \hat{\boldsymbol{\sigma}}_{t}\right)$, can be found and it yields $\tilde{\ell}\left(\hat{\boldsymbol{\theta}}_{t}, \hat{\boldsymbol{\sigma}}_{t}, \xi_{t}^{*}\right), \quad t=1,2, \ldots, G$. Hence, the maximum likelihood estimates are given by:

$$
(\hat{\boldsymbol{\theta}}, \hat{\boldsymbol{\sigma}}, \hat{\xi})=\arg \max _{\left\{\left(\hat{\boldsymbol{\theta}}_{t}, \hat{\boldsymbol{\sigma}}_{t}, \xi_{t}^{*}\right): t=1,2, \ldots, G\right\}} \tilde{\ell}\left(\hat{\boldsymbol{\theta}}_{t}, \hat{\boldsymbol{\sigma}}_{t}, \boldsymbol{\xi}_{t}^{*}\right) .
$$

To maximize the log-likelihood function in equation (6), the Olsson version (1974) of the Nelder-Mead simplex algorithm (1965) was used. The approach minimizes a function by 
construction of a simplex of points; the dimension for each corresponds to the number of parameters that must be estimated. The magnitude of the simplex is the number of parameters +1 . Maximization of $\tilde{\ell}$ was accomplished by minimizing $-\tilde{\ell}$ using the simplex algorithm. This algorithm is coded in FORTRAN, version 90.

The asymptotic variance of the estimates in $(\hat{\boldsymbol{\theta}}, \hat{\boldsymbol{\sigma}}, \hat{\xi})$ was obtained by evaluating $\mathbf{I}^{-1}(\boldsymbol{\theta}, \boldsymbol{\sigma}, \boldsymbol{\xi})$, the inverse of the observed information matrix, at $(\hat{\boldsymbol{\theta}}, \hat{\boldsymbol{\sigma}}, \hat{\xi})$. (The formula for $\mathbf{I}(\boldsymbol{\theta}, \boldsymbol{\sigma}, \boldsymbol{\xi})$ is in the Appendix.) The algorithm for computing asymptotic variances was written in $\mathrm{R}$, version 2.5.1. Because the model with one parameter to account for variability (the single$\sigma$ model) is nested within the model that accounts for variability with multiple parameters (the multiple- $\sigma$ model in equation (1)), the likelihood ratio test (LRT) can be used to test for significance of the multiple- $\sigma$ model.

Results

The proposed model in equation (1) was applied to a well-known data set extracted from a developmental toxicity study conducted at the National Toxicology Program (Tyl, et al., 1983) and set $G=1499$. The study was an experiment whereby fetal implants were injected into $131 \mathrm{CD}-1$ mice, which were subsequently randomly allocated across $g=5$ dose levels of the developmentally toxic substance diethylhexyl phthalate (DEHP). The 5 dose levels are 0 , $0.025,0.05,0.10$, and 0.15 , in units of $\%$ of DEHP in the animal diet. Animals were allocated roughly equally across dose levels. The summarized results of the experiment are in Table 1. The first and last columns of Table 1 are indicative of a threshold dose-response relationship.

The complete vector of parameters for the multiple- $\sigma$ model applied to this data is given by $\left(\theta_{1}, \theta_{2}, \theta_{3}, \xi, \sigma_{1}, \sigma_{2}, \sigma_{3}, \sigma_{4}, \sigma_{5}\right)$. Because this is a 9-parameter model, the simplex method was applied as described previously. Olsson's algorithm allows up to 20 parameters. The resulting parameter estimates are shown in Table 2 and are compared with the estimates from the original single- $\sigma$ model. Standard errors (SEs) (also shown in Table 2), were estimated by the method described. Table 2 shows that all related estimates from the two models are relatively comparable, with some noticeable bias in the estimates of the $\theta$ s. The log-likelihood estimated from (7) is -534.502 with the log-likelihood from the single- $\sigma$ model being -546.334 ; the resulting LRT statistic for the test of $H_{0}: \sigma_{i}=\sigma, i=1, \ldots, 5$ is 23.664; based on $4 d f$, the p-value is $9.326 \times 10^{-5}$, indicating significance of the multiple- $\sigma$ model.

Figure 1 shows the plot of the linear $B$ spline basis based on equation (3); based on the estimated interior knot value 0.036 , the spline basis is constructed to have linearity below and

Table 1: Summary of Results of Study of Fetal Exposure to DEHP

\begin{tabular}{|c|c|c|c|c|}
\hline $\begin{array}{c}\text { DEHP Dose (\% } \\
\text { of Animal Diet) }\end{array}$ & $\begin{array}{c}\text { Number } \\
\text { of Litters }\end{array}$ & $\begin{array}{c}\text { Total Number } \\
\text { of. Fetuses }\end{array}$ & $\begin{array}{c}\text { Number of } \\
\text { Affected Fetuses }\end{array}$ & $\begin{array}{c}\text { Proportion of } \\
\text { Affected Fetuses }\end{array}$ \\
\hline 0 & 30 & 396 & 75 & 0.189 \\
\hline 0.025 & 26 & 320 & 37 & 0.116 \\
\hline 0.05 & 26 & 319 & 80 & 0.251 \\
\hline 0.10 & 24 & 276 & 192 & 0.696 \\
\hline 0.15 & 25 & 308 & 302 & 0.981 \\
\hline
\end{tabular}




\section{LI \& HUNT}

above the knot. Figure 2 shows the plot of the logistically transformed estimated dose-response curve based on equation (1) without the random effects. This plot is indicative of two separate linear functions below and above the interior knot.

Figure 3 shows the plots of the two estimated curves from the multiple- $\sigma$ and single- $\sigma$ models, respectively. For the multiple$\sigma$ model, the interior knot 0.036 is very close to being the threshold value as the below-knot pattern follows closely to a horizontal line. For the single- $\sigma$ model, it is more of a linear pattern of decreasing slope below the knot. This degree of difference can be important as threshold estimation is crucial aspect of this analysis. Although estimate itself is relatively close, the general dose-response relationship is different and is indicative that the multiple- $\sigma$ model is more appropriate in this situation.

\section{Conclusion}

A linear B-spline threshold dose-response model was modified to include multiple parameters for modeling dose-specific response variation in developmental toxicity study data. The previous model showed only singular response variation across dose groups. Upon application of this new model, it was found that the addition of multiple parameters affected the estimates of non-variation model parameters and led to statistical significance of the new model over the prior one. The spline approach also is more robust than typical approaches that use standard regression functions which restrict the doseresponse relationship to one pattern.

Another desirable feature of using splines for fitting is that it includes models which inherently assume a threshold. The approach of using regression splines to account for threshold effects has been used recently in other fields. For example, Molinari, et al. (2001) used spline functions in place of the standard linear functions in a Cox regression analysis of survival data from several clinical trials and Bessaoud, et al. (2005) used spline functions in the logistic regression setting for analysis of clinical data. The spline approach used in this study is similar to these. Both other studies also extended their model to handle several covariates and indicated the practicality of using linear splines to estimate an interior knot as a threshold value. As they were dealing with larger data sets, both groups looked at cases of multiple knots and higher-order spline functions, although neither went past cubic splines and 3

Table 2: Estimates from the Multiple- and Single*- $\sigma$ Models

\begin{tabular}{|c|c|c|}
\hline Parameter & $\begin{array}{c}\text { Multiple- } \sigma \\
\text { Estimates }(\mathrm{SE})\end{array}$ & $\begin{array}{c}\text { Single- } \sigma \text { Estimates } \\
(\mathrm{SE})\end{array}$ \\
\hline$\theta_{1}$ & $-2.006(0.352)$ & $-2.022(0.305)$ \\
\hline$\theta_{2}$ & $-2.108(0.277)$ & $-2.530(0.357)$ \\
\hline$\theta_{3}$ & $5.519(0.831)$ & $4.668(0.490)$ \\
\hline$\xi$ & $0.036(0.006)$ & $0.033(0.007)$ \\
\hline$\sigma_{1}$ & $1.426(0.266)$ & $1.331(0.153)^{*}$ \\
\hline$\sigma_{2}$ & $0.009(0.823)$ & NA \\
\hline$\sigma_{3}$ & $0.783(0.223)$ & NA \\
\hline$\sigma_{4}$ & $2.554(0.770)$ & NA \\
\hline$\sigma_{5}$ & $1.947(0.472)$ & NA \\
\hline
\end{tabular}

*Single- $\sigma$ model has only one variability parameter 
Figure 1: The Linear $B$-spline Basis on the Dose Interval [0, 0.15], with Estimated Interior Knot $\hat{\xi}=0.036$
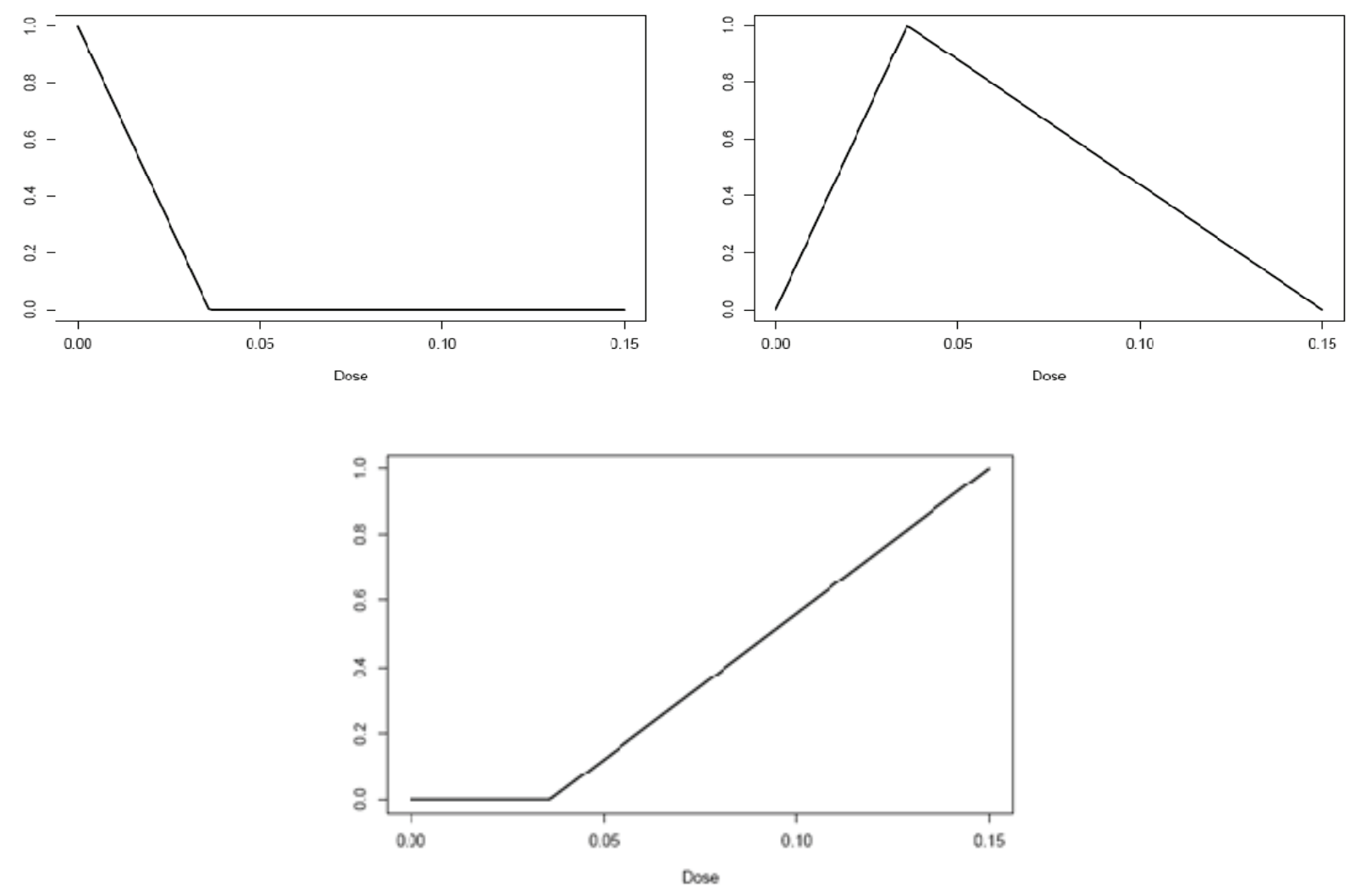

Figure 2: The Fitted Curve (solid line) and 95\% Point-Wise Confidence Interval (dashed and dotted lines) for Logit(P)

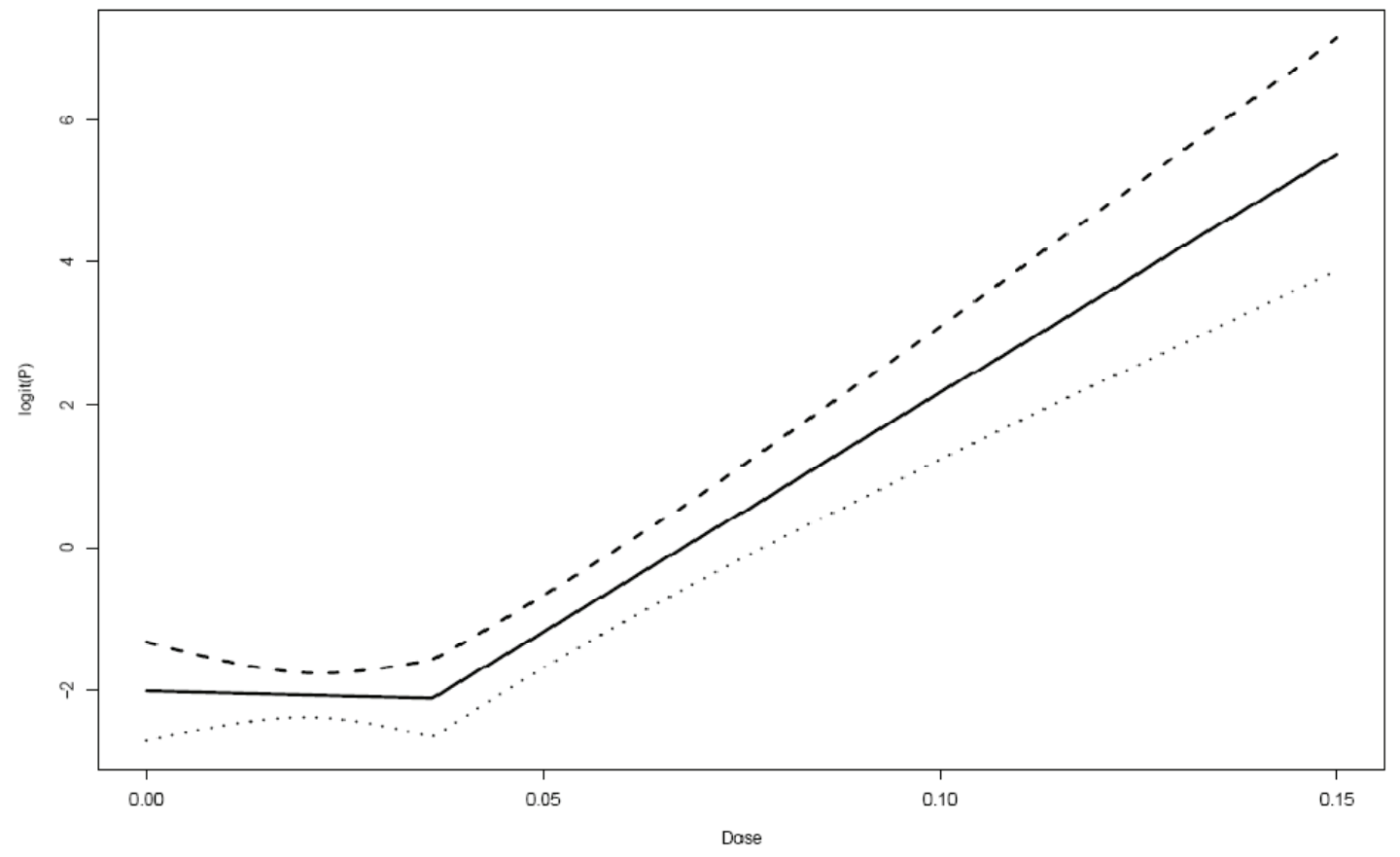




\section{LI \& HUNT}

Figure 3: Estimated Dose-Response Curves for the Multiple- $\sigma$ Model (left) and the Single- $\sigma$ Model (right)

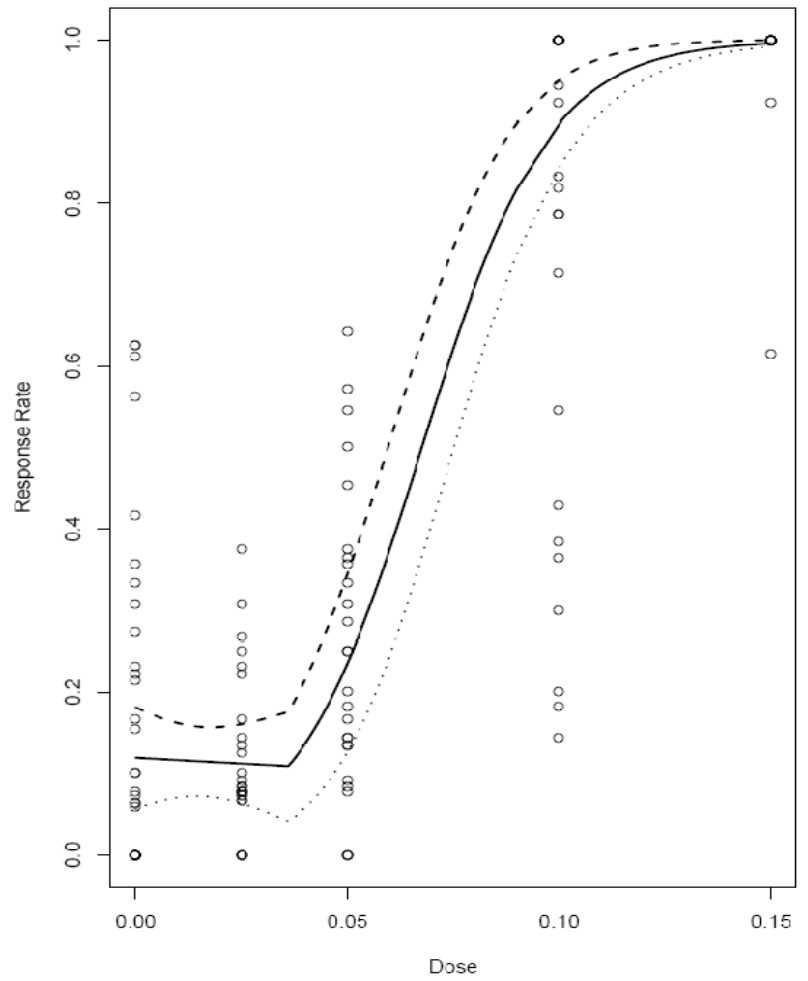

knots. Bessaoud, et al. (2005) only used up to quadratic splines and both indicated that cubic splines resulted in overfitting and that linear and quadratic seemed appropriate.

For developmental toxicity studies, the existence of a threshold is inherent in current guidelines and is accounted for in some manner during the risk assessment process, albeit indirectly (USEPA, 1991). The pure threshold model is a start in the direction of more adequately modeling these effects, yet the threshold itself has proved to be difficult to ascertain, and any threshold estimated from such a model may be specific to that data set, rather than being a universal value (Cox, 1987). The use of the splines to model behavior that is common to threshold models may help address this issue. Although the interior knot estimated in this study is not specifically the threshold dose level, the model is useful in that it identifies a change point in the direction of the dose-response pattern. It also inherently assumes threshold existence and robustly models several

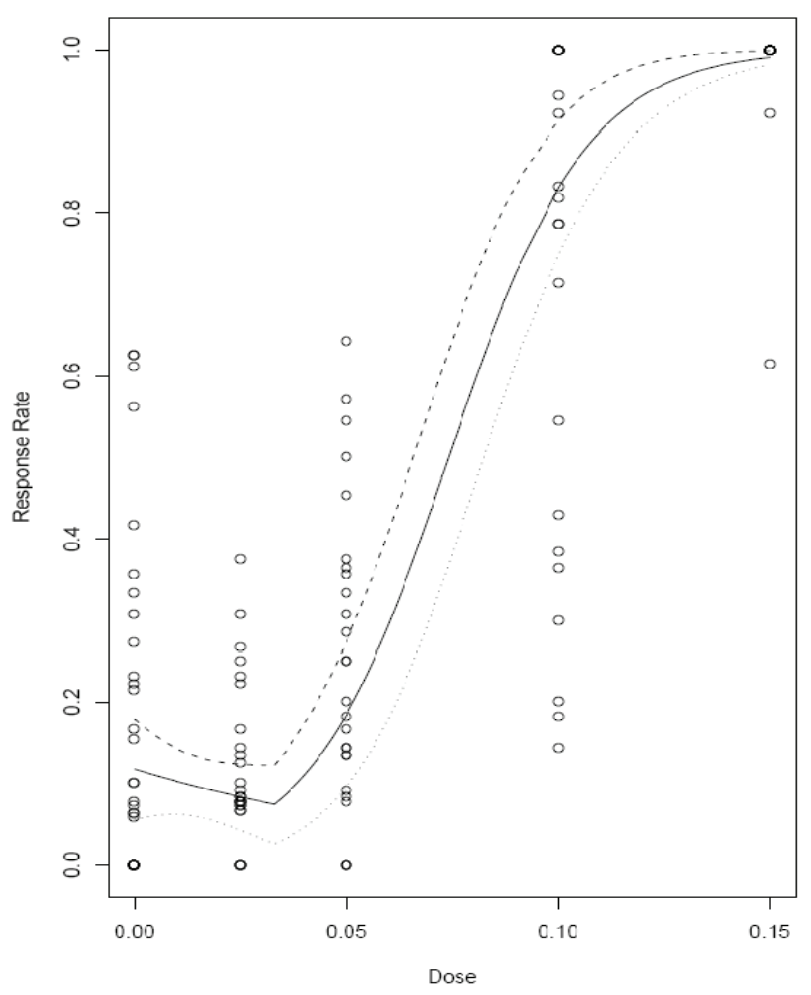

other possible dose-response patterns (Hunt \& Li, 2006).

Another advantage of the spline model is the addition of parameters to more adequately model the different degrees of response variation observed to occur across dose groups in a developmental toxicity study. This more accurately specified model improves the estimation of important parameters such as the threshold or, in the case of the spline model, the change point. As illustrated in Kupper, et al. (1986) and in Hunt and Rai (2007), the model assuming multiple parameters to model response variation leads to negligible bias, whereas under conditions of major differences in dose-specific variation the model with single parameter may lead to extremely biased estimates. Thus, the model that has multiple variation parameters is the general model that should be used. However, Hunt and Rai (2007) also showed that in cases of relatively similar variation across dose groups, the single parameter model may suffice. 


\section{SPLINE MODEL WITH DOSE-SPECIFIC RESPONSE VARIATION}

The potential for future applications in this area include the fitting of higher degree polynomials; for example, the quadratic $B$-spline might be a reasonable extension to the current linear $B$-spline approach. The most immediate advantage is the relative smoothness of the quadratic spline over the linear. However, disadvantages include overfitting. Also, the number of dose levels becomes a factor when adding additional knots into the estimation. The combination of higher order and multiple knots could result in an overly complex model for this type of data. Due to the observed dose-response pattern of the data set under investigation in this article, the linear spline model with one knot appears to provide reasonable fit.

The polynomial regression splines approach is a generally advantageous way to model data from developmental toxicity studies. Rather than requiring a direct estimation of a threshold level, it is able to fit several doseresponse curves to the data and implicitly can still indicate the existence of effects such as threshold. It is more robust than previously employed threshold models to such data (Cox, 1987; Schwartz, et al., 1995; Hunt \& Rai, 2003, 2007). Additionally, the modification of having dose-specific variation allows for an even more robust model with less biased estimates.

Acknowledgements

This work was supported by Grant Number UL1 RR024146 from the National Center for Research Resources (NCRR), a component of the National Institutes of Health (NIH), and NIH Roadmap for Medical Research. (C. S. Li). This work was partially supported by Cancer Center Support CA21765 from the National Institutes of Health, USA, and the American Lebanese Syrian Associated Charities (ALSAC) (D. L. Hunt).

\section{References}

Abramowitz, M., \& Stegun, I. A. (1972). Handbook of Mathematical Functions with Formulas, Graphs and Mathematical Tables. Washington, DC: U.S. Government Printing Office.
Bessaoud, F., Daures, J. P., \& Molinari, N. (2005). Free knot splines for logistics models and threshold selection. Computer Methods and Programs in Biomedicine, 77, 1-9.

Collett, D. (1991). Modelling binary data. Boca Raton, FL: CRC Press.

Cox, C. (1987). Threshold doseresponse models in toxicology. Biometrics, 43, 511-523.

Crump, K. S. (1984). A new method for determining allowable daily intakes. Fundamental and Applied Toxicology, 4, 854871.

De Boor, C. (2001). A Practical Guide to Splines (Revised Edition). NY: Springer.

Hunt, D. L., \& Li, C. S. (2006). A regression spline model for developmental toxicity data. Toxicological Sciences, 92, 329334.

Hunt, D. L., \& Rai, S. N. (2003). A threshold dose-response model with random effects in teratological experiments. Communications in Statistics-Theory and Methods, 32, 1439-1457.

Hunt, D. L., \& Rai, S. N. (2007). Interlitter response variability in a threshold dose-response model. Submitted to Communications in Statistics-Theory and Methods.

Kupper, L. L., Portier, C., Hogan, M. D. \& Yamamoto, E. (1986). The impact of litter effects on dose-response modeling in teratology. Biometrics, 42, 85-98.

Li, C. S., \& Hunt, D. (2004). Regression splines for threshold selection with application to a random-effects logistic dose-response model. Computational Statistics and Data Analysis, 46, 1-9.

Molinari, N., Daures, J. P., \& Durand, J. F. (2001) Regression splines for threshold selection in survival data analysis. Statistics in Medicine, 20, 237-247.

Nelder, J. A., \& Mead, R. (1965). A simplex method for function minimization. Computer Journal, 7, 308-313.

Olsson, D. M. (1974). A sequential simplex program for solving minimization problems. Statistical Computer Program, 6, 5357. 


\section{LI \& HUNT}

Ramsay, J. O. (1988). Monotone regression splines in action. Statistical Science, 3, 425-461.

Ryan, L. (2000). Statistical issues in toxicology. Journal of the American Statistical Association, 95, 304-308.

Schwartz, P., Gennings, C. \& Chinchilli, V. (1995). Threshold models for combination data from reproductive and developmental experiments. Journal of the American Statistical Association, 90, 862-870.
Tyl, R. W., Jones-Price, C., Marr, M. C. \& Kimmel., C. A. (1983). Teratological evaluation of diethylhexyl phthalate (CAS No. 117-81-7) in CD-1 mice. Final Study Report for NCTR/NTP Contract NO. 222-80-2031 9(c). NTIS NO. PB85105674, National Technical Information Service, Springfield, VA.

USEPA. (1991). Guidelines for developmental toxicity risk assessment. Federal Register, 56, 63798-63826.

Appendix

To obtain the observed information matrix $\mathbf{I}(\boldsymbol{\theta}, \boldsymbol{\sigma}, \boldsymbol{\xi})$, the second-order partial derivatives of $\tilde{\ell}(\boldsymbol{\theta}, \boldsymbol{\sigma}, \boldsymbol{\xi})$ in equation (7) must be calculated with respect to $(\boldsymbol{\theta}, \boldsymbol{\sigma}, \boldsymbol{\xi})$ and $\tilde{\ell}(\boldsymbol{\theta}, \boldsymbol{\sigma}, \boldsymbol{\xi})$ as:

$\tilde{\ell}(\boldsymbol{\theta}, \boldsymbol{\sigma}, \xi)=\sum_{i=1}^{g} \sum_{j=1}^{m_{i}} \log \left(H_{i j}\right)$.

Here, $H_{i j}=\sum_{l=1}^{q} a_{l} R_{i l}^{x_{i j}} \bar{R}_{i l}^{\bar{x}_{i j}}, R_{i l}=\frac{\exp \left(\eta_{i}+\sigma_{i} b_{l} \sqrt{2}\right)}{1+\exp \left(\eta_{i}+\sigma_{i} b_{l} \sqrt{2}\right)}, \eta_{i}=\sum_{k=1}^{3} \theta_{k} B_{k, 2}\left(d_{i}, \xi\right)=\mathbf{B}_{2}\left(d_{i}, \xi\right) \boldsymbol{\theta}$,

$\bar{R}_{i l}=1-R_{i l}$, and $\bar{x}_{i j}=n_{i j}-x_{i j}$. The first-order partial derivatives of $\tilde{\ell}(\boldsymbol{\theta}, \boldsymbol{\sigma}, \xi)$ with respect to $(\boldsymbol{\theta}, \boldsymbol{\sigma}, \xi)$ are:

$$
\begin{aligned}
& \frac{\partial \tilde{\ell}(\boldsymbol{\theta}, \boldsymbol{\sigma}, \boldsymbol{\xi})}{\partial \boldsymbol{\theta}}= \frac{\partial}{\partial \boldsymbol{\theta}} \sum_{i=1}^{g} \sum_{j=1}^{m_{i}} \log \left(H_{i j}\right) \\
&= \sum_{i=1}^{g} \sum_{j=1}^{m_{i}} H_{i j}^{-1} \frac{\partial H_{i j}}{\partial \boldsymbol{\theta}} \\
&= \sum_{i=1}^{g} \sum_{j=1}^{m_{i}} H_{i j}^{-1}\left\{\sum_{l=1}^{q} a_{l}\left(x_{i j} R_{i l}^{x_{i j}} \bar{R}_{i l}^{\bar{x}_{i j}+1}-\bar{x}_{i j} R_{i l}^{x_{i j}+1} \bar{R}_{i l}^{\bar{x}_{i j}}\right) \frac{\partial \eta_{i}}{\partial \boldsymbol{\theta}}\right\}, \\
& \frac{\partial \tilde{\ell}(\boldsymbol{\theta}, \boldsymbol{\sigma}, \boldsymbol{\xi})=}{\partial \sigma_{r}}=\frac{\partial}{\partial \sigma_{r}} \sum_{i=1}^{g} \sum_{j=1}^{m_{i}} \log \left(H_{i j}\right) \\
&=\sum_{i=1}^{g} \sum_{j=1}^{m_{i}} H_{i j}^{-1} \frac{\partial H_{i j}}{\partial \sigma_{r}} I_{(r=i)} \\
&=\sum_{i=1}^{g} \sum_{j=1}^{m_{i}} H_{i j}^{-1}\left\{\sum_{l=1}^{q} a\left(x_{i j} R_{i l}^{x_{i j}} \bar{R}_{i l}^{\bar{x}_{i j}+1}-\bar{x}_{i j} R_{i l}^{x_{i j}+1} \bar{R}_{i l}^{\bar{x}_{i j}}\right) b_{l} \sqrt{2} I_{(r=i)}\right\}, r=1, \ldots, g,
\end{aligned}
$$


and

Appendix (continued)

$$
\begin{aligned}
\frac{\partial \tilde{\ell}(\boldsymbol{\theta}, \boldsymbol{\sigma}, \xi)}{\partial \xi}= & \frac{\partial}{\partial \xi} \sum_{i=1}^{g} \sum_{j=1}^{m_{i}} \log \left(H_{i j}\right) \\
& =\sum_{i=1}^{g} \sum_{j=1}^{m_{i}} H_{i j}^{-1} \frac{\partial H_{i j}}{\partial \xi} \\
& =\sum_{i=1}^{g} \sum_{j=1}^{m_{i}} H_{i j}^{-1}\left\{\sum_{l=1}^{q} a_{l}\left(x_{i j} R_{i l}^{x_{i j}} \bar{R}_{i l}^{\bar{x}_{i j}+1}-\bar{x}_{i j} R_{i l}^{x_{i j}+1} \bar{R}_{i l}^{\bar{x}_{i j}}\right) \frac{\partial \eta_{i}}{\partial \xi}\right\}
\end{aligned}
$$

where

$$
\begin{gathered}
\frac{\partial \eta_{i}}{\partial \boldsymbol{\theta}^{T}}=\frac{\partial}{\partial \boldsymbol{\theta}^{T}} \sum_{k=1}^{3} \theta_{k} B_{k, 2}\left(d_{i}, \xi\right)=\left[B_{1,2}\left(d_{i}, \xi\right), B_{2,2}\left(d_{i}, \xi\right), B_{3,2}\left(d_{i}, \xi\right)\right]=\mathbf{B}_{2}\left(d_{i}, \xi\right), \text { and } \\
\frac{\partial \eta_{i}}{\partial \xi}=\theta_{1} \frac{d_{i}-d_{1}}{\left(\xi-d_{1}\right)^{2}} I_{\left(d_{i}<\xi\right)}+\theta_{2}\left(\frac{d_{1}-d_{i}}{\left(\xi-d_{1}\right)^{2}} I_{\left(d_{i}<\xi\right)}+\frac{d_{g}-d_{i}}{\left(d_{g}-\xi\right)^{2}} I_{\left(d_{i}>\xi\right)}\right)+\theta_{3} \frac{d_{i}-d_{g}}{\left(d_{g}-\xi\right)^{2}} I_{\left(d_{i}>\xi\right)}
\end{gathered}
$$

As a result, the components of $\mathbf{I}(\boldsymbol{\theta}, \boldsymbol{\sigma}, \boldsymbol{\xi})$ are given, respectively, as follows:

$$
\begin{aligned}
& -\frac{\partial^{2} \tilde{\ell}(\boldsymbol{\theta}, \boldsymbol{\sigma}, \boldsymbol{\xi})}{\partial \boldsymbol{\theta} \partial \boldsymbol{\theta}^{T}}=-\sum_{i=1}^{g} \sum_{j=1}^{m_{i}}\left\{\left(\frac{\partial H_{i j}^{-1}}{\partial \boldsymbol{\theta}}\right) \frac{\partial H_{i j}}{\partial \boldsymbol{\theta}^{T}}+H_{i j}^{-1} \frac{\partial^{2} H_{i j}}{\partial \boldsymbol{\theta} \partial \boldsymbol{\theta}^{T}}\right\} \\
& =-\sum_{i=1}^{g} \sum_{j=1}^{m_{i}} H_{i j}^{-1}\left\{\sum_{l=1}^{q} a_{l}\left(x_{i j}^{2} R_{i l}^{x_{i j}} \bar{R}_{i l}^{\bar{x}_{i j}+2}-\left(x_{i j}+2 x_{i j} \bar{x}_{i j}+\bar{x}_{i j}\right) R_{i l}^{x_{i j}+1} \bar{R}_{i l}^{\bar{x}_{i j}+1}+\bar{x}_{i j}^{2} R_{i l}^{x_{i j}+2} \bar{R}_{i l}^{\bar{x}_{i j}}\right) \frac{\partial \eta_{i}}{\partial \boldsymbol{\theta}} \frac{\partial \eta_{i}}{\partial \boldsymbol{\theta}^{T}}\right\} \\
& +\sum_{i=1}^{g} \sum_{j=1}^{m_{i}} H_{i j}^{-2}\left[\sum_{l=1}^{q} a_{l}\left(x_{i j} R_{i l}^{x_{i j}} \bar{R}_{i l}^{\bar{x}_{i j}+1}-\bar{x}_{i j} R_{i l}^{x_{i j}+1} \bar{R}_{i l}^{\bar{x}_{i j}}\right) \frac{\partial \eta_{i}}{\partial \boldsymbol{\theta}}\right]\left[\sum_{l=1}^{q} a_{l}\left(x_{i j} R_{i l}^{x_{i j}} \bar{R}_{i l}^{\bar{x}_{i j}+1}-\bar{x}_{i j} R_{i l}^{x_{i j}+1} \bar{R}_{i l}^{\bar{x}_{i j}}\right) \frac{\partial \eta_{i}}{\partial \boldsymbol{\theta}^{T}}\right], \\
& -\frac{\partial^{2} \tilde{\ell}(\boldsymbol{\theta}, \boldsymbol{\sigma}, \xi)}{\partial \xi \partial \boldsymbol{\theta}^{T}}=-\sum_{i=1}^{g} \sum_{j=1}^{m_{i}}\left\{\left(\frac{\partial H_{i j}^{-1}}{\partial \xi}\right) \frac{\partial H_{i j}}{\partial \boldsymbol{\theta}^{T}}+H_{i j}^{-1} \frac{\partial^{2} H_{i j}}{\partial \xi \partial \boldsymbol{\theta}^{T}}\right\} \\
& =-\sum_{i=1}^{g} \sum_{j=1}^{m_{i}} H_{i j}^{-1} \frac{\partial^{2} H_{i j}}{\partial \xi \partial \boldsymbol{\theta}^{T}}+\sum_{i=1}^{g} \sum_{j=1}^{m_{i}} H_{i j}^{-2} \frac{\partial H_{i j}}{\partial \xi} \frac{\partial H_{i j}}{\partial \boldsymbol{\theta}^{T}} \\
& =-\sum_{i=1}^{g} \sum_{j=1}^{m_{i}} H_{i j}^{-1}\left\{\sum_{l=1}^{q} a_{l}\left(x_{i j}^{2} R_{i l}^{x_{i j}} \bar{R}_{i l}^{\bar{x}_{i j}+2}-\left(x_{i j}+2 x_{i j} \bar{x}_{i j}+\bar{x}_{i j}\right) R_{i l}^{x_{i j}+1} \bar{R}_{i l}^{\bar{x}_{i j}+1}+\bar{x}_{i j}^{2} R_{i l}^{x_{i j}+2} \bar{R}_{i l}^{\bar{x}_{i j}}\right) \frac{\partial \eta_{i}}{\partial \xi} \frac{\partial \eta_{i}}{\partial \boldsymbol{\theta}^{T}}\right\} \\
& -\sum_{i=1}^{g} \sum_{j=1}^{m_{i}} H_{i j}^{-1}\left[\sum_{l=1}^{q} a_{l}\left(x_{i j} R_{i l}^{x_{i j}} \bar{R}_{i l}^{\bar{x}_{i j}+1}-\bar{x}_{i j} R_{i l}^{x_{i j}+1} \bar{R}_{i l}^{\bar{x}_{i j}}\right) \frac{\partial^{2} \eta_{i}}{\partial \xi \partial \boldsymbol{\theta}^{T}}\right] \\
& +\sum_{i=1}^{g} \sum_{j=1}^{m_{i}} H_{i j}^{-2}\left[\sum_{l=1}^{q} a_{l}\left(x_{i j} R_{i l}^{x_{i j}} \bar{R}_{i l}^{\bar{x}_{i j}+1}-\bar{x}_{i j} R_{i l}^{x_{i j}+1} \bar{R}_{i l}^{\bar{x}_{i j}}\right) \frac{\partial \eta_{i}}{\partial \xi}\right]\left[\sum_{l=1}^{q} a_{l}\left(x_{i j} R_{i l}^{x_{i j}} \bar{R}_{i l}^{\bar{x}_{i j}+1}-\bar{x}_{i j} R_{i l}^{x_{i j}+1} \bar{R}_{i l}^{\bar{x}_{i j}}\right) \frac{\partial \eta_{i}}{\partial \boldsymbol{\theta}^{T}}\right],
\end{aligned}
$$




\section{LI \& HUNT}

Appendix (continued)

$$
\begin{aligned}
& -\frac{\partial^{2} \tilde{\ell}(\boldsymbol{\theta}, \boldsymbol{\sigma}, \xi)}{\partial \sigma_{r} \partial \sigma_{s}}=-\sum_{i=1}^{g} \sum_{j=1}^{m_{i}}\left\{\left(\frac{\partial H_{i j}^{-1}}{\partial \sigma_{r}}\right) \frac{\partial H_{i j}}{\partial \sigma_{s}}+H_{i j}^{-1} \frac{\partial^{2} H_{i j}}{\partial \sigma_{r} \partial \sigma_{s}}\right\} \\
& =-\sum_{i=1}^{g} \sum_{j=1}^{m_{i}}\left(\frac{\partial H_{i j}^{-1}}{\partial \sigma_{r}}\right)\left[\sum_{l=1}^{q} a_{l}\left(x_{i j} R_{i l}^{x_{i j}} \bar{R}_{i l}^{\bar{x}_{i l}+1}-\bar{x}_{i j} R_{i l}^{x_{i j}+1} \bar{R}_{i l}^{\bar{x}_{i j}}\right) b_{l} \sqrt{2} I_{(s=i)}\right] \\
& -\sum_{i=1}^{g} \sum_{j=1}^{m_{i}} H_{i j}^{-1}\left\{\sum_{l=1}^{q} a_{l}\left[\frac{\partial}{\partial \sigma_{r}}\left(x_{i j} R_{i l}^{x_{i j}} \bar{R}_{i l}^{\bar{x}_{i j}+1}-\bar{x}_{i j} R_{i l}^{x_{i j}+1} \bar{R}_{i l}^{\bar{x}_{i j}}\right)\right] b_{l} \sqrt{2} I_{(s=i)}\right\} \\
& =\sum_{i=1}^{g} \sum_{j=1}^{m_{i}} H_{i j}^{-2}\left[\sum_{l=1}^{q} a_{l}\left(x_{i j} R_{i l}^{x_{i j}} \bar{R}_{i l}^{\bar{x}_{i j}+1}-\bar{x}_{i j} R_{i l}^{x_{i j}+1} \bar{R}_{i l}^{\bar{x}_{i j}}\right) b_{l} \sqrt{2} I_{(r=i)}\right]\left[\sum_{l=1}^{q} a_{l}\left(x_{i j} R_{i l}^{x_{i j}} \bar{R}_{i l}^{\bar{x}_{i j}+1}-\bar{x}_{i j} R_{i l}^{x_{i j}+1} \bar{R}_{i l}^{\bar{x}_{i j}}\right) b_{l} \sqrt{2} I_{(s=i)}\right] \\
& -\sum_{i=1}^{g} \sum_{j=1}^{m_{i}} H_{i j}^{-1}\left\{\sum_{l=1}^{q} a_{l}\left[x_{i j}^{2} R_{i l}^{x_{i j}} \bar{R}_{i l}^{\bar{x}_{i j}+2}-\left(x_{i j}+2 x_{i j} \bar{x}_{i j}+\bar{x}_{i j}\right) R_{i l}^{x_{i j}+1} \bar{R}_{i l}^{\bar{x}_{i j}+1}+\bar{x}_{i j}^{2} R_{i l}^{x_{i j}+2} \bar{R}_{i l}^{\bar{x}_{i j}}\right] 2 b_{l}^{2} I_{(r=i)} I_{(s=i)}\right\} \\
& \int-\sum_{j=1}^{m_{i j}} H_{i j}^{-1}\left\{\sum_{l=1}^{q} a_{l}\left[x_{r j}^{2} R_{r l}^{x_{r j}} \bar{R}_{r l}^{\bar{x}_{j l}+2}-\left(x_{r j}+2 x_{r j} \bar{x}_{r j}+\bar{x}_{r j}\right) R_{r l}^{x_{i j}+1} \bar{R}_{r l}^{\bar{x}_{i j}+1}+\bar{x}_{r j}^{2} R_{r l}^{x_{i j}+2} \bar{R}_{r l}^{\bar{x}_{r j}}\right] 2 b_{l}^{2}\right\} \\
& =\left\{\begin{array}{rr}
\quad+\sum_{j=1}^{m_{i}} H_{i j}^{-2}\left[\sum_{l=1}^{q} a_{l}\left(x_{r j} R_{r l}^{x_{i j}} \bar{R}_{r l}^{\bar{x}_{r l}+1}-\bar{x}_{r j} R_{r l}^{x_{r j}+1} \bar{R}_{r l}^{\bar{x}_{r j}}\right) b_{l} \sqrt{2}\right]^{2}, s=r=i \\
0, \quad s \neq r ; s=r \neq i,
\end{array}\right.
\end{aligned}
$$

$$
\begin{aligned}
& -\frac{\partial^{2} \tilde{\ell}(\boldsymbol{\theta}, \boldsymbol{\sigma}, \xi)}{\partial \xi \partial \sigma_{r}}=-\sum_{i=1}^{g} \sum_{j=1}^{m_{i}}\left\{\left(\frac{\partial H_{i j}^{-1}}{\partial \xi}\right) \frac{\partial H_{i j}}{\partial \sigma_{r}}+H_{i j}^{-1} \frac{\partial^{2} H_{i j}}{\partial \xi \partial \sigma_{r}}\right\} \\
& =-\sum_{i=1}^{g} \sum_{j=1}^{m_{i}} H_{i j}^{-1}\left\{\sum_{l=1}^{q} a_{l}\left(x_{i j}^{2} R_{i l}^{x_{i j}} \bar{R}_{i l}^{\bar{x}_{i j}+2}-\left(x_{i j}+2 x_{i j} \bar{x}_{i j}+\bar{x}_{i j}\right) R_{i l}^{x_{i j}+1} \bar{R}_{i l}^{\bar{x}_{i j}+1}+\bar{x}_{i j}^{2} R_{i l}^{x_{i j}+2} \bar{R}_{i l}^{\bar{x}_{i j}}\right) \sqrt{2} b_{l} I_{(r=i)} \frac{\partial \eta_{i}}{\partial \xi}\right\} \\
& +\sum_{i=1}^{g} \sum_{j=1}^{m_{i}} H_{i j}^{-2}\left[\sum_{l=1}^{q} a_{l}\left(x_{i j} R_{i l}^{x_{i j}} \bar{R}_{i l}^{\bar{x}_{i j}+1}-\bar{x}_{i j} R_{i l}^{x_{i j}+1} \bar{R}_{i l}^{\bar{x}_{i j}}\right) \frac{\partial \eta_{i}}{\partial \xi}\right]\left[\sum_{l=1}^{q} a_{l}\left(x_{i j} R_{i l}^{x_{i j}} \bar{R}_{i l}^{\bar{x}_{i j}+1}-\bar{x}_{i j} R_{i l}^{x_{i j}+1} \bar{R}_{i l}^{\bar{x}_{i j}}\right) \sqrt{2} b_{l} I_{(r=i)} .\right. \\
& \int-\sum_{j=1}^{m_{i}} H_{i j}^{-1}\left\{\sum_{l=1}^{q} a_{l}\left(x_{i j}^{2} R_{i l}^{x_{i j}} \bar{R}_{i l}^{\bar{x}_{i j}+2}-\left(x_{i j}+2 x_{i j} \bar{x}_{i j}+\bar{x}_{i j}\right) R_{i l}^{x_{i j}+1} \bar{R}_{i l}^{\bar{x}_{i j}+1}+\bar{x}_{i j}^{2} R_{i l}^{x_{i j}+2} \bar{R}_{i l}^{\bar{x}_{i j}}\right) \sqrt{2} b_{l} \frac{\partial \eta_{i}}{\partial \xi}\right\} \\
& =\left\{+\sum_{j=1}^{m_{i}} H_{i j}^{-2}\left[\sum_{l=1}^{q} a_{l}\left(x_{i j} R_{i l}^{x_{i j}} \bar{R}_{i l}^{\bar{x}_{i j}+1}-\bar{x}_{i j} R_{i l}^{x_{i j}+1} \bar{R}_{i l}^{\bar{x}_{i j}}\right) \frac{\partial \eta_{i}}{\partial \xi}\right]\left[\sum_{l=1}^{q} a_{l}\left(x_{i j} R_{i l}^{x_{i j}} \bar{R}_{i l}^{\bar{x}_{i j}+1}-\bar{x}_{i j} R_{i l}^{x_{i j}+1} \bar{R}_{i l}^{\bar{x}_{i j}}\right) \sqrt{2} b_{l}\right], r=i\right. \\
& 0 \text {, } \\
& , r \neq i \text {, }
\end{aligned}
$$




\section{Appendix (continued)}

$$
\begin{aligned}
-\frac{\partial^{2} \tilde{\ell}(\boldsymbol{\theta}, \boldsymbol{\sigma}, \xi)}{\partial \xi^{2}}= & -\sum_{i=1}^{g} \sum_{j=1}^{m_{i}}\left\{\left(\frac{\partial H_{i j}^{-1}}{\partial \xi}\right) \frac{\partial H_{i j}}{\partial \xi}+H_{i j}^{-1} \frac{\partial^{2} H_{i j}}{\partial \xi^{2}}\right\} \\
= & -\sum_{i=1}^{g} \sum_{j=1}^{m_{i}} H_{i j}^{-1}\left\{\sum_{l=1}^{q} a_{l}\left[x_{i j}^{2} R_{i l}^{x_{i j}} \bar{R}_{i l}^{\bar{x}_{i j}+2}-\left(x_{i j}+2 x_{i j} \bar{x}_{i j}+\bar{x}_{i j}\right) R_{i l}^{x_{i j}+1} \bar{R}_{i l}^{\bar{x}_{i j}+1}+\bar{x}_{i j}^{2} R_{i l}^{x_{i j}+2} \bar{R}_{i l}^{\bar{x}_{i j}}\right]\left(\frac{\partial \eta_{i}}{\partial \xi}\right)^{2}\right\} \\
& -\sum_{i=1}^{g} \sum_{j=1}^{m_{i}} H_{i j}^{-1}\left[\sum_{l=1}^{q} a_{l}\left(x_{i j} R_{i l}^{x_{i j}} \bar{R}_{i l}^{\bar{x}_{i j}+1}-\bar{x}_{i j} R_{i l}^{x_{i j}+1} \bar{R}_{i l}^{\bar{x}_{i j}}\right) \frac{\partial^{2} \eta_{i}}{\partial \xi^{2}}\right] \\
& +\sum_{i=1}^{g} \sum_{j=1}^{m_{i}} H_{i j}^{-2}\left[\sum_{l=1}^{q} a_{l}\left(x_{i j} R_{i l}^{x_{i j}} \bar{R}_{i l}^{\bar{x}_{i j}+1}-\bar{x}_{i j} R_{i l}^{x_{i j}+1} \bar{R}_{i l}^{\bar{x}_{i j}}\right) \frac{\partial \eta_{i}}{\partial \xi}\right]^{2},
\end{aligned}
$$

where

$$
\frac{\partial^{2} \eta_{i}}{\partial \xi^{2}}=2 \theta_{1} \frac{d_{1}-d_{i}}{\left(\xi-d_{1}\right)^{3}} I_{\left(d_{i}<\xi\right)}+2 \theta_{2}\left(\frac{d_{i}-d_{1}}{\left(\xi-d_{1}\right)^{3}} I_{\left(d_{i}<\xi\right)}+\frac{d_{g}-d_{i}}{\left(d_{g}-\xi\right)^{3}} I_{\left(d_{i}>\xi\right)}\right)+2 \theta_{3} \frac{d_{i}-d_{g}}{\left(d_{g}-\xi\right)^{3}} I_{\left(d_{i}>\xi\right)}
$$

and

$$
\frac{\partial^{2} \eta_{i}}{\partial \xi \partial \boldsymbol{\theta}^{T}}=\left[\frac{d_{i}-d_{1}}{\left(\xi-d_{1}\right)^{2}} I_{\left(d_{i}<\xi\right)}, \frac{d_{1}-d_{i}}{\left(\xi-d_{1}\right)^{2}} I_{\left(d_{i}<\xi\right)}+\frac{d_{g}-d_{i}}{\left(d_{g}-\xi\right)^{2}} I_{\left(d_{i}>\xi\right)}, \frac{d_{i}-d_{g}}{\left(d_{g}-\xi\right)^{2}} I_{\left(d_{i}>\xi\right)}\right] .
$$

